John Carroll University

Carroll Collected

\title{
Molecular dynamics simulations of apo and holo forms of fatty acid binding protein 5 and cellular retinoic acid binding protein II reveal highly mobile protein, retinoic acid ligand, and water molecules
}

Nathanael H. Hunter

John Carroll University

Blair C. Bakula

John Carroll University

Chrystal D. Bruce

John Carroll University, cbruce@jcu.edu

Follow this and additional works at: https://collected.jcu.edu/fac_bib_2018

Part of the Chemistry Commons

\section{Recommended Citation}

Hunter, Nathanael H.; Bakula, Blair C.; and Bruce, Chrystal D., "Molecular dynamics simulations of apo and holo forms of fatty acid binding protein 5 and cellular retinoic acid binding protein II reveal highly mobile protein, retinoic acid ligand, and water molecules" (2018). 2018 Faculty Bibliography. 17.

https://collected.jcu.edu/fac_bib_2018/17

This Article is brought to you for free and open access by the Faculty Bibliographies Community Homepage at Carroll Collected. It has been accepted for inclusion in 2018 Faculty Bibliography by an authorized administrator of Carroll Collected. For more information, please contact connell@jcu.edu. 


\title{
Molecular dynamics simulations of apo and holo forms of fatty acid binding protein 5 and cellular retinoic acid binding protein II reveal highly mobile protein, retinoic acid ligand, and water molecules
}

\author{
Nathanael H. Hunter, Blair C. Bakula and Chrystal D. Bruce* (1) \\ Department of Chemistry, John Carroll University, University Heights, OH, USA
}

\begin{abstract}
Structural and dynamic properties from a series of $300 \mathrm{~ns}$ molecular dynamics, MD, simulations of two intracellular lipid binding proteins, iLBPs, (Fatty Acid Binding Protein 5, FABP5, and Cellular Retinoic Acid Binding Protein II, CRABP-II) in both the apo form and when bound with retinoic acid reveal a high degree of protein and ligand flexibility. The ratio of FABP5 to CRABP-II in a cell may determine whether it undergoes natural apoptosis or unrestricted cell growth in the presence of retinoic acid. As a result, FABP5 is a promising target for cancer therapy. The MD simulations presented here reveal distinct differences in the two proteins and provide insight into the binding mechanism. CRABP-II is a much larger, more flexible protein that closes upon ligand binding, where FABP5 transitions to an open state in the holo form. The traditional understanding obtained from crystal structures of the gap between two $\beta$-sheets of the $\beta$-barrel common to iLBPs and the $\alpha$-helix cap that forms the portal to the binding pocket is insufficient for describing protein conformation (open vs. closed) or ligand entry and exit. When the high degree of mobility between multiple conformations of both the ligand and protein are examined via MD simulation, a new mode of ligand motion that improves understanding of binding dynamics is revealed.
\end{abstract}

Keywords: molecular dynamics; lipid binding protein; fatty acid binding protein; cellular retinoic acid binding protein; ligand binding mechanism

\section{Introduction}

Intracellular lipid binding proteins (iLBPs) have long been recognized as critical in the transport and improved bio-availability of lipid ligands via reversible, non-covalent binding. iLBPs have recently received attention as potential therapeutic targets for a number of diseases including cancer and diabetes because of their role in signaling pathways (Armstrong, Goswami, Griffin, Noy, \& Ortlund, 2014; Glatz, 2015; Storch \& Thumser, 2010). One such target is the class of iLBPs known as Fatty Acid Binding Proteins (FABP). The family of FABPs is comprised of nine separate proteins of 14$15 \mathrm{kDa}$ found in a wide variety of human tissues. Despite their ubiquitous presence in the body, FABP function is highly tissue-specific (Atshaves et al., 2010; Ayers, Nedrow, Gillilan, \& Noy, 2007; Cao et al., 2008; De Leon et al., 1996; Gillilan, Ayers, \& Noy, 2007; Liu, Almaguel, Bu, De Leon, \& De Leon, 2008; Newberry et al., 2003; Newberry, Xie, Kennedy, Luo, \& Davidson, 2006; Owada, 2008; Schug, Berry, Shaw, Travis, \& Noy, 2007a; Schug et al., 2008; Storch \& Thumser, 2010; Tan et al., 2002; Xie, Newberry, Kennedy, Luo, \& Davidson, 2009). Within the FABP family, the sequence homology is only 20-70\% (Armstrong et al., 2014; Storch \& Thumser, 2010). However, the tertiary structures of all FABPs are similar, containing 10 antiparallel $\beta$ sheets $(\beta A-\beta J)$ to create a $\beta$-barrel structure and a helix-turn-helix motif ( $\alpha \mathrm{I}-\alpha \mathrm{II})$ (Balendiran et al., 2000; Hohoff, Börchers, Rüstow, Spener, \& Van Tilbeurgh, 1999; Lucke, Gutierrez-Gonzalez, \& Hamilton, 2003; Marr et al., 2006; Ruskamo et al., 2014; Schaap, Van der Vusse, \& Glatz, 2002). The region between $\beta \mathrm{D}$ and $\beta \mathrm{E}$ is separated by distances greater than that required for hydrogen bonding to occur and is referred to as the 'gap,' shown in blue in Figure 1. Access to the relatively large binding pocket is proposed to occur via the 'portal,' which is located near the helix-turn-helix region of the protein and is shown in green in Figure 1. However, molecular dynamics studies of other lipid-binding proteins indicate that surface binding may play a role in ligand binding in some proteins in this family (Levin et al., 2010; Long, Mu, \& Yang, 2009).

The focus of the work presented here is the fatty acid binding protein FABP5 (sometimes referred to as E-FABP or KFABP). FABP5 is important as a potential target for cancer therapy, because it binds retinoic acid, 


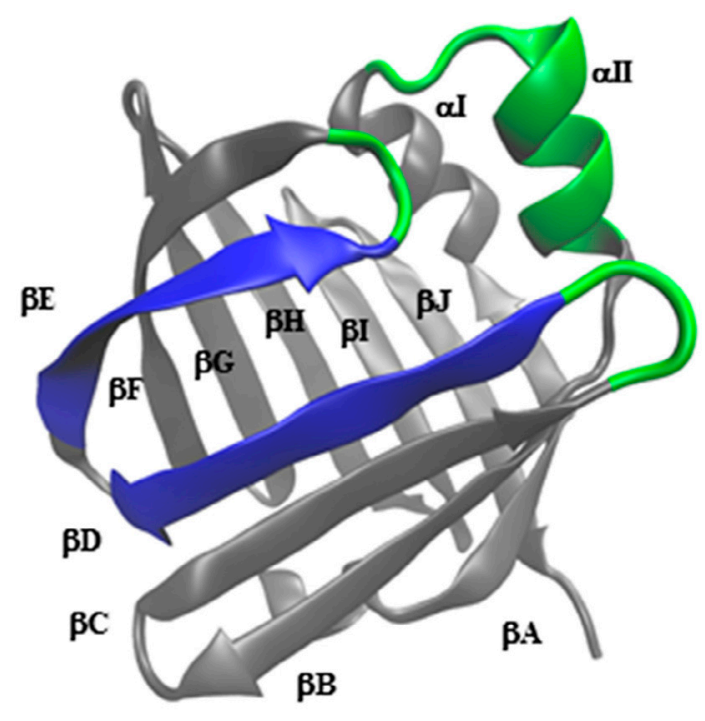

Figure 1. The starting structure of the simulation of FABP5 Only with the ten $\beta$-sheets $(\beta \mathrm{A}-\beta \mathrm{J})$ and two $\alpha$-helices that are common to the entire FABP5 family and CRABP-II labeled.

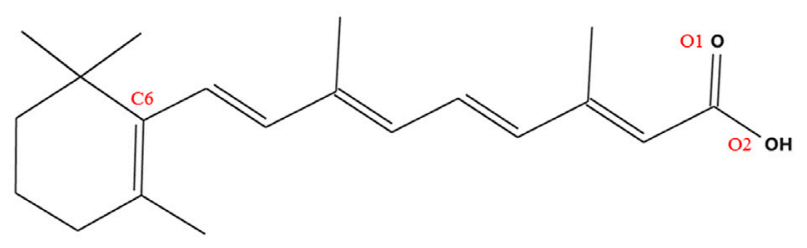

Figure 2. Retinoic acid with atoms important in later discussion labeled.

RA (Figure 2), a metabolite of Vitamin A, and subsequently activates the peroxisome proliferator-activated receptor $\beta / \delta$ (PPAR $\beta / \delta$ resulting in transcription of genes relating to cell growth (Armstrong et al., 2014; Schug et al., 2007a; Schug, Berry, Shaw, Travis, \& Noy, 2007b; Schug et al., 2008). Normally, retinoic acid (RA) is bound by Cellular Retinoic Acid Binding Protein II (CRABP-II) and the Retinoic Acid Receptor (RAR) is activated which signals apoptosis to occur. Usually, the RAR pathway is dominant, in part because the binding affinity of retinoic acid is higher for the CRABP-II/RAR pathway (Kds .1-.2 nm) (Dong, Ruuska, Levinthal, \& Noy, 1999; Sussman \& de Lera, 2005) than for the FABP5/ PPAR $\beta / \delta$ pathway (Kds $10-50 \mathrm{~nm}$ ) (Schug et al., 2007a; Tan et al., 2002). However, in cells that have high amounts of FABP5, activation of the PPAR $\beta / \delta$ pathway occurs, resulting in unwanted cell proliferation (Schug et al., 2007a; Schug et al., 2008; Storch \& Thumser, 2010). The ratio of CRAPB-II to FABP5 determines whether cell proliferation or growth inhibition will be favored (Schug et al., 2008). CRABP-II acts as a tumor suppressor when there is a sufficiently high ratio of CRABP-II to FABP5. Although much experimental work has been done to understand how these proteins impact cancer cell growth or inhibition, there are still many questions as evidenced by recent reviews that highlight how much is still unknown about what ratio of CRABP-II to FABP5 is required for the RAR route to dominate (Peters, Gonzalez, \& Müller, 2015) and the actual role of PPAR $\beta / \delta$ pathway in human cancer (Mueller, 2016).

CRABP-II and FABP5 have the same three-dimensional $\beta$-barrel structure even though they only have a $32 \%$ sequence homology, similar to that within the FABP5 family. Figure S1 shows an overlay of CRABPII and FABP5 to demonstrate their similar three-dimensional structure, and Table S1 shows the full sequence of each protein. While a number of experimental and computational studies have been performed on fatty acid binding proteins and other intracellular lipid binding proteins, including recent studies on FABP3 (Matsuoka, Sugiyama, Murata, \& Matsuoka, 2015) and FABP4, (Chen, Wang, \& Zhu, 2014; Li, Li, \& Dong, 2014; Zhou et al., 2016) simulations of FABP5 and CRABP-II are lacking. FABP5 is relevant as a potential therapeutic target for cells where the ratio of FABP5 to CRABP-II is high. In this study, we discuss results from a series of $300 \mathrm{~ns}$ molecular dynamics simulations: FABP5 alone, CRABP-II alone, FABP5 bound with retinoic acid, and three trajectories of CRABP-II bound with retinoic acid. Comparison between ligand-containing (holo) and ligand-free (apo) forms of each protein reveal structural and dynamic differences that provide insight into binding and ligand design.

\section{Methods}

Starting structures for each protein were obtained from the protein data bank (pdb id 1B56 for FABP5 and pdb id 2CBS for CRABPII). The FABP5 protein from the pdb has 133 residues (numbered 3-135) while the CRABP-II protein has 137 residues. All ligand and water molecules in the protein crystal structure were deleted. The retinoic acid ligand structure was drawn in Spartan 14 , saved in .pdb format, and docked into the binding pocket of the corresponding protein using Autodock Vina. The lowest energy conformation was saved as the starting point for subsequent molecular dynamics simulations. As confirmation of the quality of the docked structure, the location of the docked retinoic acid (RA) was compared to that of the structurally similar ligand in the CRABP-II crystal structure. The overlap, as shown in Figure S2, indicates that Autodock Vina provided reasonable starting locations for the ligands in these proteins. Antechamber was used to determine the gaff force field parameters for the retinoic acid ligand, which was subsequently saved in a .mol2 file. The xleap program in 
AMBER 14 program was then used to add ions as needed (CRABP-II has a -2 charge, FABP5 is neutral) and solvate the complex with TIP3P waters using a truncated octahedron having a minimum distance of $10 \AA$ from the protein to the edge of the box, resulting in box sizes approximately $61 \AA \times 61 \AA \times 61 \AA$. Each solvated complex was then subjected to minimization followed by 50 ps of heating under NVT conditions to reach $300 \mathrm{~K}$, $50 \mathrm{ps}$ of an NPT simulation at $300 \mathrm{~K}$ to equilibrate the density of the solvent, and a $1 \mathrm{~ns}$ NPT equilibration prior to the $300 \mathrm{~ns}$ production run. A 2 fs time step was applied, periodic boundary conditions were used, and all bonds involving hydrogen were constrained using the SHAKE algorithm. Snapshots were recorded every $25 \mathrm{ps}$ for analysis. The production runs were conducted using the CUDA version of PMEMD to maximize GPU acceleration of sander using NVIDIA GPU (Salomon-Ferrer, Götz, Poole, Le Grand, \& Walker, 2013). Analysis of the production runs were conducted using cpptraj within AmberTools 15 (Roe, Cheatham, \& Cheatham, 2013), MMPBSA.py script in Amber (Miller et al., 2012), and VMD 1.9.1 (Humphrey, Dalke, \& Schulten, 1996).

\section{Results and discussion}

\subsection{Protein global structure and flexibility}

All four systems show a stable protein (FABP5 -only and CRABP-II-only) or protein/ligand complex (FABP5+RA and CRABP-II+RA) throughout the $300 \mathrm{~ns}$ simulation. In the latter simulations, the ligand remains in the binding pocket region, although the ligand exhibits a high degree of flexibility as will be discussed below. There are times when the portal and gap open and close as the retinoic acid ligand moves around in the large binding pocket, but it does not fully exit the protein in the $300 \mathrm{~ns}$ simulations. To examine the global structure of the proteins, the average root-mean-squared deviations, RMSD, and radius of gyration, $R_{g}$, for each of the 4 systems were calculated using Amber15 Tools and are shown in Table 1. The time-dependent behavior of these values for each simulation is available in the Supplementary Material. The RMSD fluctuates somewhat for each of the four systems consistent with the findings for FABP4 of Li et al. (2014) It is apparent from the consistently larger values of the averages and standard deviations of both the RMSD and $R_{g}$ that CRABP-II is a larger, more flexible protein than FABP5. This result is consistent with reports that a similar iLBP, CRABP-I, also exhibits flexible, dynamic behavior (Ferrolino, Zhuravleva, Budyak, Krishnan, \& Gierasch, 2013).

The ability of the protein to open and close allows water and ligands to enter and exit the protein. To quantify the amount of time each protein was in either an open or closed state, the open conformation was defined as one where the radius of gyration was greater than .5 $\AA$ above the $R_{g}$ of the crystal structure, consistent with the defintion of Matsuoka et al. for FABP3 (Matsuoka et al., 2015). The radius of gyration of the starting crystal structures were $14.2 \AA$ for FABP5 (pdb id 1B56) and $14.6 \AA$ for CRABP-II ( $\mathrm{pdb}$ id 2CBS). Therefore, the protein was considered open when the radius of gyration was greater than $14.7 \AA$ for FABP5 and greater than $15.1 \AA$ for CRABP-II. See Figure 3 for open and closed conformations of FABP5. The average radius of gyration in every simulation is larger than the $R_{g}$ of the crystal structure both with and without bound ligand. As expected, these proteins appear to be more flexible and open in solution. Table 1 shows that the percentage of time the protein stays open changes in the presence of ligand, but the direction of the change differs for CRABP-II and FABP5. In the case of CRABP-II, the protein is in the closed confirmation more often in the holo form, while for FABP5, the protein is in the open confirmation more often in the holo form, although in general, FABP5 is in the closed form far more often. An analysis of the conformational states of FABP4 shows that the closed conformation is dominant for the apo form and that FABP4 is more open upon ligand binding, consistent with the findings here for FABP5 (Li et al., 2014).

The flexiblity of the protein backbone in the apo and holo states can be further evaluated by the root meansquared fluctuations (RMSF) of $\mathrm{C} \alpha$ atoms in each residue as shown in Figure 4. The secondary structures for each protein are shown at the top of Figure 4 and are separated by protein, because the residue numbers that comprise the $\alpha$-helices and $\beta$-sheets differ slighly between FABP5 and CRABP-II. RMSF values are less than one for atoms within the $\beta$-sheets, indicating very little positional fluctuation. The helix-turn-helix region of

Table 1. Summary of RMSD, $R_{g}$, and percentage of time the protein spends in an open conformation for each simulation.

\begin{tabular}{lcccc}
\hline & $\begin{array}{c}\text { FABP5 } \\
\text { only }\end{array}$ & FABP5+RA & $\begin{array}{c}\text { CRABP-II } \\
\text { only }\end{array}$ & CRABPII+RA \\
\hline RMSD $(\AA)$ & $2.89 \pm .30$ & $2.54 \pm .30$ & $3.54 \pm .55$ & $2.78 \pm .56$ \\
$R_{g}(\AA)$ & $14.42 \pm .14$ & $14.56 \pm .11$ & $15.28 \pm .17$ & $15.13 \pm .18$ \\
$\%$ OPEN & 5.4 & 11.2 & 85.7 & 55.5 \\
\hline
\end{tabular}



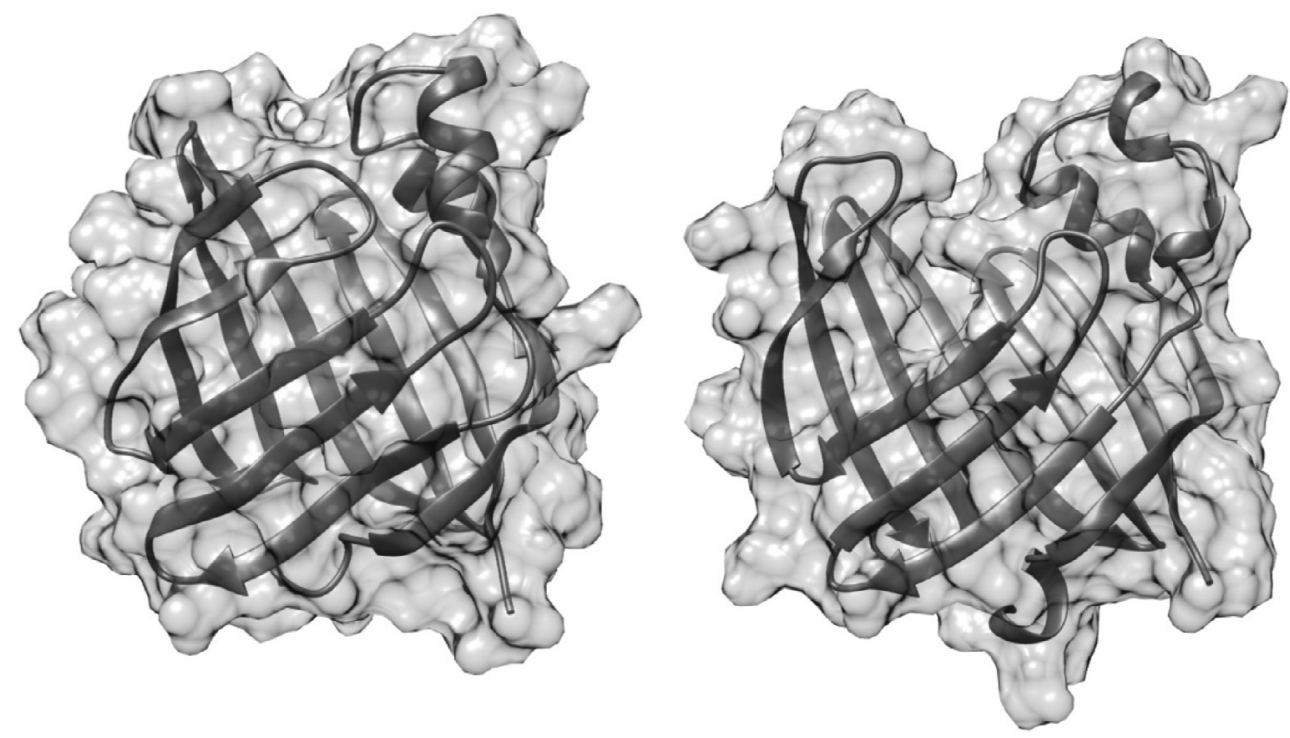

Figure 3. Surface of FABP5 Only at $80 \%$ transparency in the closed (left, $t=10 \mathrm{~ns}$ ) and open (right, $t=60 \mathrm{~ns}$ ) conformation. In the open conformation, defined as having a radius of gyration greater than $14.7 \AA$ for FABP5, there is significant access to the binding pocket for ligand and solvent ingress or egress.

the proteins showed larger fluctuations indicating larger mobility than the atoms in the $\beta$-sheets. These results are consistent with the hypothesis that the residues in the helix-turn-helix region form the portal that opens (and closes) to allow ligands to enter the binding pocket. Regions of larger RMSF values also appear in the loops between $\beta$-sheets, particularly those facing the portal region of each protein. Visual inspection of the trajectories supports the conclusion that the loops, particularly those near the portal region, experience much motion during the simulation.

To further understand the motion indicated by the $R_{g}$ and RMSF calculations, a series of distances were monitored over the course of the simulation and are shown in Figure 5 with averages given in Table 2. To monitor the gap region, distances between $\beta \mathrm{D}$ (Phe 65 in FABP5 and Ile 63 in CRABP-II) and $\beta E$ (Glu 75 for FABP5 and Glu 73 for CRABP-II) residues were monitored. To follow changes in the portal region, distances between residues in the $\beta \mathrm{E} / \beta \mathrm{F}$ loop (Gly 36 for FABP5 and Ala 36 in CRABP-II) and $\alpha$ II (Leu 60 for FABP5 and Val 58 for CRABP-II), referred to as Portal 1, and between the $\alpha 1 /$ $\alpha$ II loop (Val 28 for FABP5 and Gly 23 in CRABP-II) and $\beta E / \beta F$ loop (Ala 78 for FABP5 and Val 76 in CRABP-II), referred to as Portal 2, were followed over the course of all simulations. Additionally, the distance between residues in the $\alpha \mathrm{I} / \alpha \mathrm{II}$ loop and the $\beta \mathrm{G} / \beta \mathrm{H}$ loop (Asp 101 for FABP-5 and Gly 102 in CRABP-II), referred to as Loop $\mathrm{G}-\mathrm{H}$, were monitored. These distances were selected rather than a single distance as in the analyis of FABP4 by Li et al. (2014) so that a clear picture of the motion of loops near the portal could be examined.
Comparing the time dependence of the radius of gyration (Figure S4) to the distances in Figure 5, it appears that CRABP-II oscillates between open and closed forms in both the apo and holo form, while FABP5 is open at times, but is primarily in the closed conformation both with and without the RA ligand. It is also clear that the Gap, Portal 1, Portal 2, and Loop G-H motions are independent of one another and fluctuate throughout the simulation. From visual inspection of the trajectories, either loop can turn out into the solvent in both the apo or holo form. It is also interesting to note that at times, the $\alpha 1 / \alpha \mathrm{II}$ loop and the $\beta \mathrm{G} / \beta \mathrm{H}$ loop are within $9 \AA$ and at others they are more than $20 \AA$ apart. This certainly adds to the discussion of where and how ligands enter and exit the binding pocket. Although briefly mentioned by $\mathrm{Li}$ et al., very little attention has been paid to the other loops near the portal region in the literature (Li et al., 2014). With the large distance fluctuations of these loops, this may be another potential location for water or ligand ingress and egress from the binding pocket.

\subsection{Ligand motion in FABP5}

A lingering question in the literature on lipid binding proteins is the mechanism of ligand binding. Clearly this is an important question when evaluating drug targets and for understanding relative binding affinities. RA has been shown to bind to both CRABP-II and FABP5 with reasonable Kds $(.1-.2 \mathrm{~nm}$ for CRABP-II [Dong et al., 1999; Sussman \& de Lera, 2005] and 10-50 nm for FABP5 [Schug et al., 2007a; Tan et al., 2002]). Crystal 

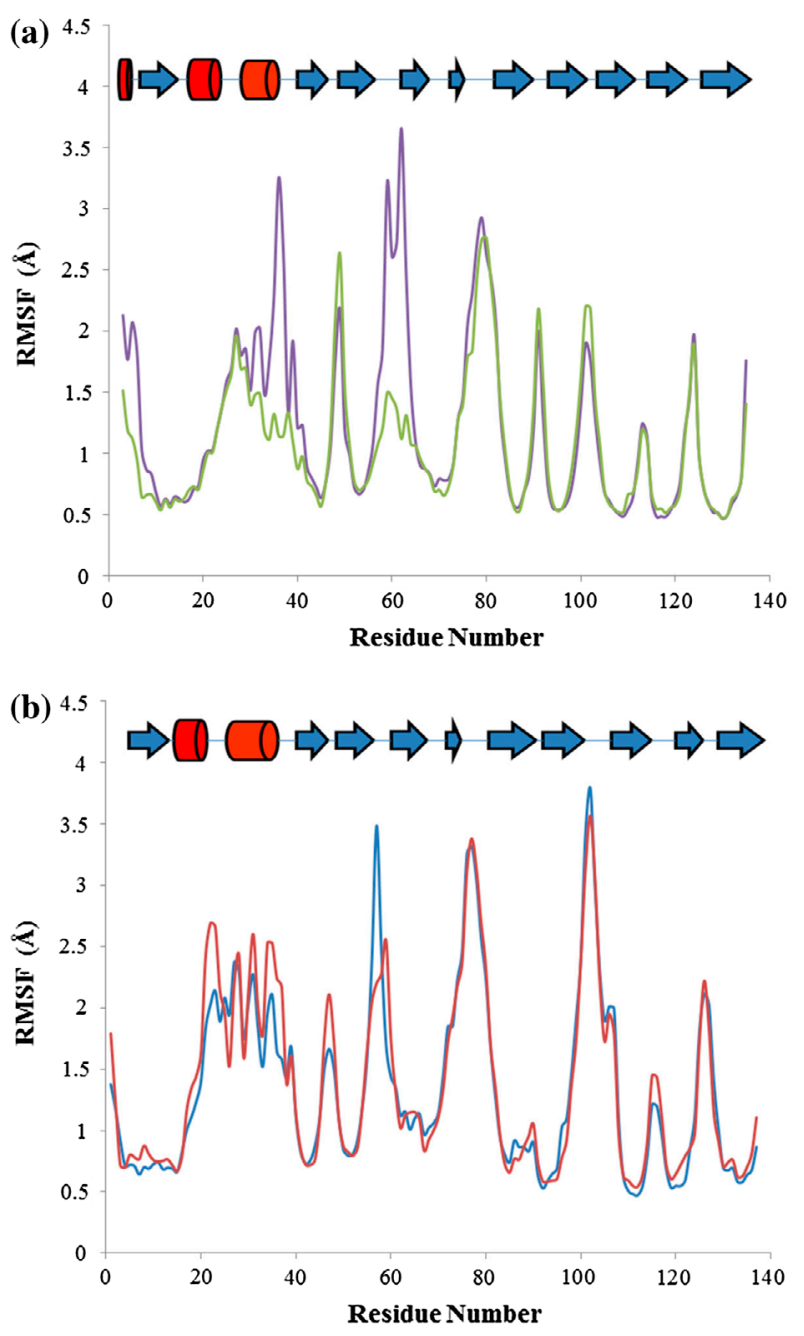

Figure 4. RMSF values of the $\mathrm{C} \alpha$ atoms in the protein for (a) FABP5 Only (purple) and FABP5+RA (green) and (b) CRABP-II Only (blue) and CRABPII+RA (red). The protein secondary stuctures are shown above each figure.

structures can give some insight into why that is the case, but they only provide a partial story. MD simulations provide insight into the dynamic behavior of protein-ligand interactions that are unable to be seen in the solid state. In solution, neither the protein nor the ligand are rigid. The crystal structures do however, highlight the importance of certain residues in binding. In the case of FABP5, Arg 129, and Tyr 131, both of which are part of $\beta \mathrm{J}$, form hydrogen bonds with the carboxylic acid functional group when crystalized with a fatty acid (Van Tilbeurgh, Hohoff, Borchers, \& Spener, 1999). While these hydrogen bonds are observed in some of the frames of the FABP5+RA simulation, more often hydrogen bonds are observed between RA and Arg 109, which is part of $\beta \mathrm{H}$. In the starting structure of FABP5+RA, RA O1 is equidistant from Arg 109 and Arg 129 as shown in Figure S5. In the solution-phase simulation here, RA apparently prefers to form hydrogen bonds with Arg 109 rather than Arg 129. During the course of the simulation, RA O1 forms a hydrogen bond with Arg $109 \mathrm{HE}$ (see Figures 1 and S7 for atom labeling scheme for RA and Arg.) in $47 \%$ of the recorded frames while RA O2 forms a hydrogen bond with Arg $109 \mathrm{HH} 12$ in $41 \%$ of the recorded frames. Li et al. also observe conformational modes in which certain ligands will lose contact with residues that are conserved in the crystal structures of holo forms of FABP4 (Li et al., 2014).

Additional insight into the motion of the FABP5+RA complex is revealed through close inspection of the time dependence of the gap and portal distances (Figure 5). Even though the radius of gyration calculations indicate that the protein is in the closed conformation nearly $90 \%$ of the time in the holo form, the portal and gap can open, sometimes widely. To evaluate this motion, the distance between $\mathrm{O} 1$ of retinoic acid and Arg $109 \mathrm{NE}$ (the hydrogen bond mentioned in the previous paragraph) and RA C6 and the $\alpha 1 / \alpha 2$ loop using V28 C $\alpha$ ) were monitored. The former distance indicates how deep the ligand is in the binding pocket while the latter provides insight in hyrophobic interactions between the ligand and the protein.

In the region between 58-65 ns, the retinoic acid appears to lose hydrogen bond contact with Arg 109, but the hydrohobic region protein loop to RA C6 distance decreases, so the retinoic acid is not leaving the binding pocket. Snapshots at 60,140 , and $300 \mathrm{~ns}$ are shown in Figure 7. Around $60 \mathrm{~ns}$, the gap (the distance between $\beta \mathrm{D}$ and $\beta \mathrm{E})$ is very large, the hydrogen bond between RA and Arg 109 is absent, but the hydrophobic interactions between the RA and the protein are still present. The ligand is simply oriented differently in the binding pocket as it explores that available space. The snapshot at $140 \mathrm{~ns}$ was selected because Figure S4 and Figure 5 show an apparent conformational change shortly before 150 ns where Portal 1 decreases and Portal 2 increases. In this situation, the changes in portal separation correspond with a decrease in the gap size. At $140 \mathrm{~ns}$, the gap is still relatively large, while after $150 \mathrm{~ns}$, it has returned to approximately where it was at the beginning of the production run and remains there, with normal fluctuations, throughout the simulation. The $300 \mathrm{~ns}$ snapshot in Figure 7 shows the portal and gap are both relatively closed.

While RA remains bound to FABP5 during the course of the simulations (see Figure S6 for images at $50 \mathrm{~ns}$ intervals), it certainly explores a large area of the binding pocket and forces a wide gap at points during the simulation. Lipid binding proteins are known to be particularly promiscuous, binding a wide variety of structurally different ligands, only some of which activate the protein (Armstrong et al., 2014; Ayers et al., 2007; Xu, Bernlohr, \& Banaszak, 1993). The large binding pocket, 

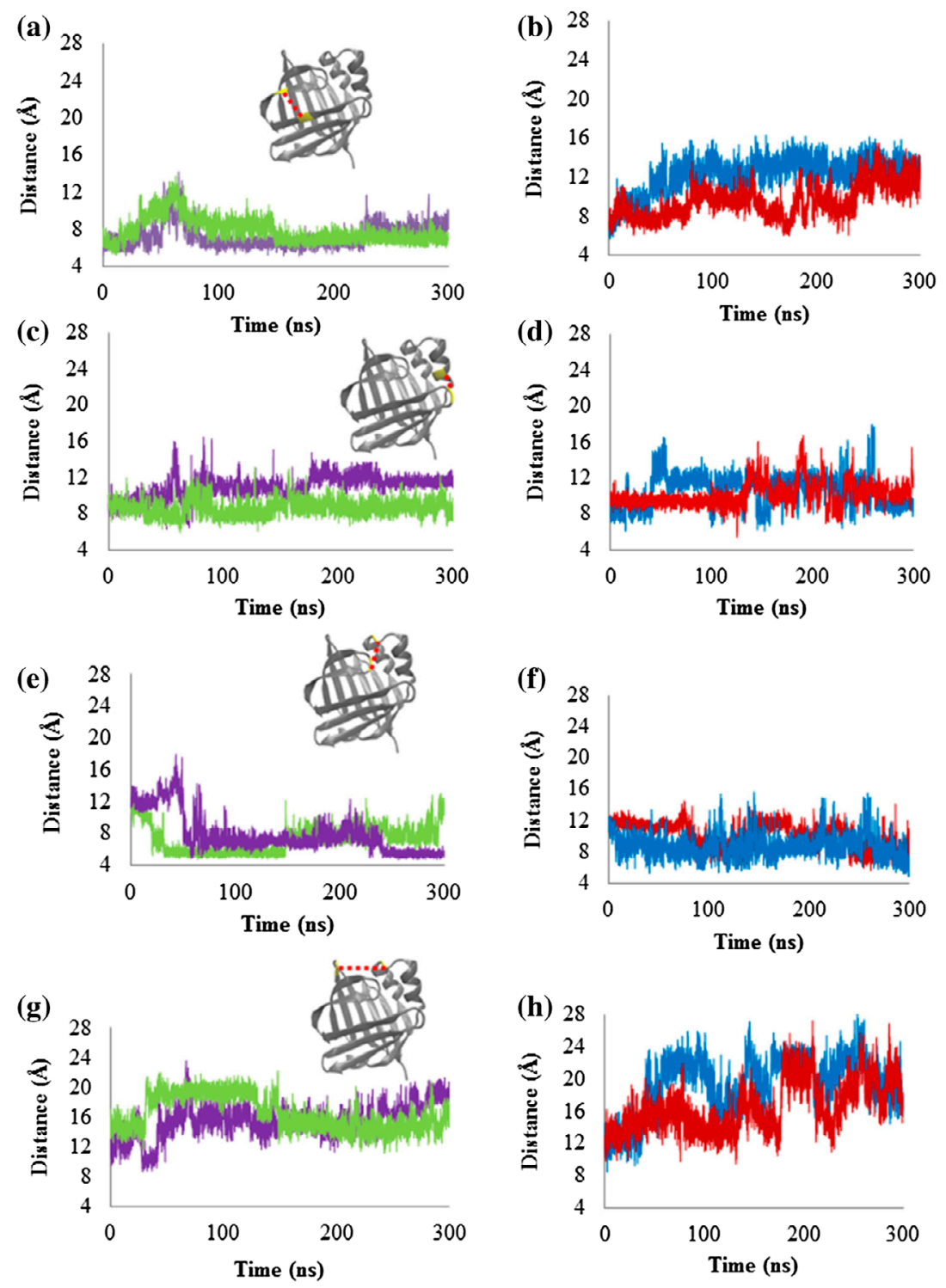

Figure 5. Distances monitored over the course of the simulation to follow the Gap (a,b), Portal 1 (c,d), Portal 2 (e,f), and Loop G-H to helix-turn-helix (g,h) distances for FABP5 Only (purple), FABP5+RA (green), CRABP-II Only (blue), and CRABPII+RA (red). Images of the protein residues being monitored are in yellow with the distance indicated by dashed red lines for each monitored distance are shown on the FABP5 plots. Locations are similar for CRABP-II, although the exact residues numbers differ and are given in the text.

Table 2. Average distances for the gap ( $\beta \mathrm{D}-\beta \mathrm{E}$ separation), Portal 1 (separation of $\beta \mathrm{E} / \beta \mathrm{F}$ loop and $\alpha \mathrm{II}$ ), Portal 2 (separation of $\alpha 1 / \alpha$ II loop and $\beta \mathrm{E} / \beta \mathrm{F}$ loop), and Loop $\mathrm{G}-\mathrm{H}$ regions (separation of $\alpha 1 / \alpha \mathrm{II}$ loop and $\beta \mathrm{G} / \beta \mathrm{H}$ loop).

\begin{tabular}{lrrrr}
\hline & FABP5 only & FABP5+RA & CRABP-II only & CRABP-II+RA \\
\hline Gap $(\AA)$ & $7.33 \pm 1.17$ & $7.87 \pm 1.35$ & $12.20 \pm 1.71$ & $9.55 \pm 1.64$ \\
Portal 1 $(\AA)$ & $8.79 \pm 1.21$ & $8.67 \pm .90$ & $7.64 \pm 1.78$ & $10.17 \pm 1.33$ \\
Portal 2 $(\AA)$ & $7.80 \pm 2.47$ & $7.15 \pm 1.70$ & $8.77 \pm 1.43$ & $10.14 \pm 1.59$ \\
Loop G-H $(\AA)$ & $15.47 \pm 2.22$ & $16.44 \pm 2.33$ & $19.28 \pm 3.47$ & $16.42 \pm 3.08$ \\
\hline
\end{tabular}


which for some iLBPs can accommodate two or three ligands, and the ability of the ligand(s) to explore many areas of the binding pocket may contribute to the low selectivity of lipid binding proteins.

For a more global view of the relative motion between the ligand and protein, the distances between their respective centers of mass was calculated to be $7.36 \pm .69 \AA$. The magnitude of the standard deviation indicates that the ligand and protein have fairly consistent separation throughout the simulation but the ligand does have some mobility, consistent with other distance monitoring reported here. To determine which protein residues interacted most favorably with the RA ligand, pairwise per-residue free energy decomposition MMGBSA calculations were performed on 400 snapshots from 50 to $250 \mathrm{~ns}$. The results of these calculations, which are best used for comparison of relative energy contributions (Genheden \& Ryde, 2015), indicate that the residues that provide the most favorable ligand-protein interactions are Arg 109, Lys 61, Thr 56, Leu 32, Pro 41, Arg 129, shown in Figure 8. Their relative contributions to the free energy are available in Table S2. It is clear that both hydrophobic and hydrophilic interactions are important to binding.

\subsection{Ligand motion in CRABP-II}

While FABP5 shows an increase in the amount of time spent in the open conformation when RA is bound as seen in Table 1, CRABP-II shows a decrease. The CRABP-II protein is closed during $45.5 \%$ of the holo simulation compared to only $14.3 \%$ of the time in the apo form. CRABP-II is a larger, more open protein than FABP5. As a result, the bound ligand has more mobility. The RA center of mass to CRABP-II center of mass distance was $11.75 \pm 1.76 \AA$, which is much larger than for FABP5. To further evaluate the mobility of RA in CRABP-II, the following distances were monitored, similar to those monitored for FABP5: (1) RA O1 to Met 123 SD to follow how deep the ligand was in the binding pocket, (2) RA C6 - Val $24 \mathrm{C} \alpha$ to determine ligand-portal interactions. Arg 111 in CRABP-II corresponds to Arg 109 in FABP5 and shows the same pattern as Met 123, except with larger distance, so Met 123 was used for clarity. The time dependence of these ligand-protein separations are shown in Figure 6. Note that no hydrogen bonding occurs between Arg 111 in CRABP-II and RA, unlike between FABP5 Arg 109 and RA. MM-GBSA pairwise per-residue free energy decomposition calculations reveal that RA interacts favorably with Arg 59, Ala 32, Leu 28, and Val 76 during the simulation. These residues are shown in Figure 9 and their contribution to the free energy of binding is given in Table S3.
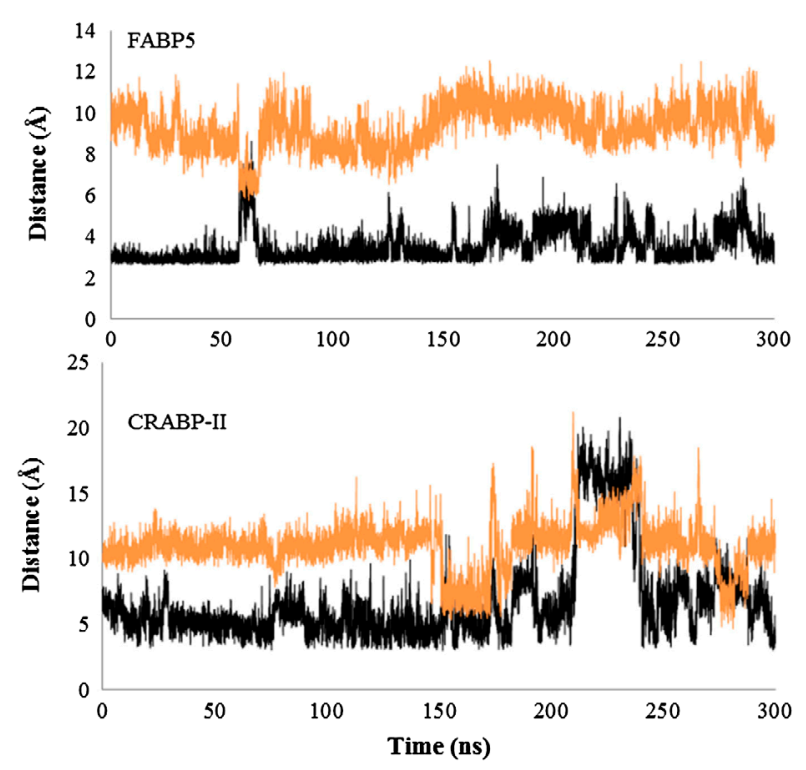

Figure 6. Distance monitoring of ligand to interior of protein via RA 01 to FABP5 Arg 109 NE (top, yellow) and to CRABP-II Met 123 SD (bottom, yellow) and to portal via RA C6 to FABP5 Val $28 \mathrm{C} \alpha$ (top, yellow) and to CRABP-II Gly $23 \mathrm{C} \alpha$ (bottom, black). RA atom numbering is shown in Figure 2.

While the distance monitoring (Figure 5) and radius of gyration (Figure S4) calculations for the global behavior of CRABP-II indicates relative overall stability of the complex, close investigation of the ligand motion as shown in Figure 6 reveals a major change in conformation of the ligand during the simulation that is not apparent from Figure 5 alone. Figure 10 shows snapshots of the complex at 50,100,150,200,230, and $250 \mathrm{~ns}$.

Around $208 \mathrm{~ns}$, the ligand makes the transition from having the carboxylic acid buried in the binding pocket to being close to the portal as shown in Figure 11(a). The RA ligand continues to rotate into the position shown in Figure 10(e); however, it does not fully exit and re-enter the binding pocket. When the carboxylic acid functional group transitions back into the binding pocket around $240 \mathrm{~ns}$, it can form hydrogen bonds with Arg 132 (See Figure S8). The RA ligand remains in the orientation shown in Figure 10(f) for the remainder of the $300 \mathrm{~ns}$ simulation. Li et al. observe what may be the beginning of this rotation with a long chain fatty acid ligand in their MD studies of FABP4, and they postulate a potential alternative opening for ligand entry and exit between the $\beta \mathrm{E} / \beta \mathrm{F}$ loop and the $\beta \mathrm{G} / \beta \mathrm{H}$ loop while the equivalent of Portal 1 is closed. They do not, however, observe this opening with larger, bulkier ligands, which extend into solvent through the portal when they protrude from the protein at all. We only observe this complete rotation of RA out of the binding pocket in 


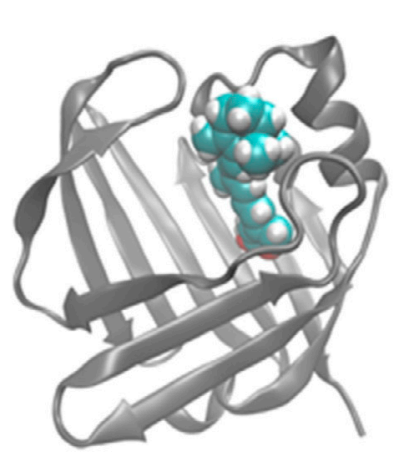

a) $60 \mathrm{~ns}$

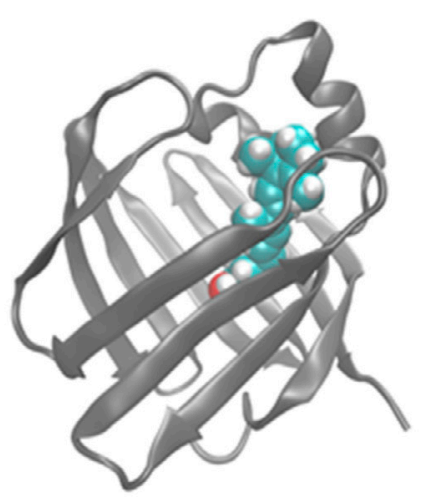

b) $140 \mathrm{~ns}$

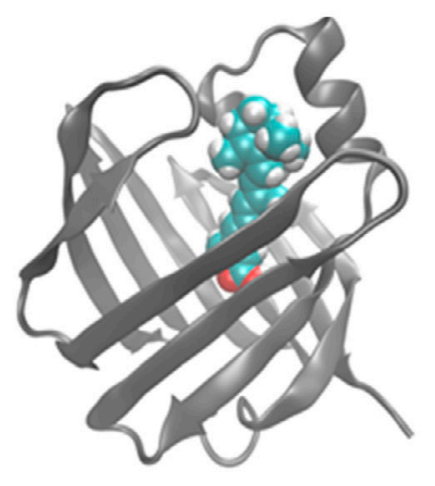

c) $300 \mathrm{~ns}$

Figure 7. FABP5+RA at $60 \mathrm{~ns}$ (left), $140 \mathrm{~ns}$ (center) and at $300 \mathrm{~ns}$ (right).

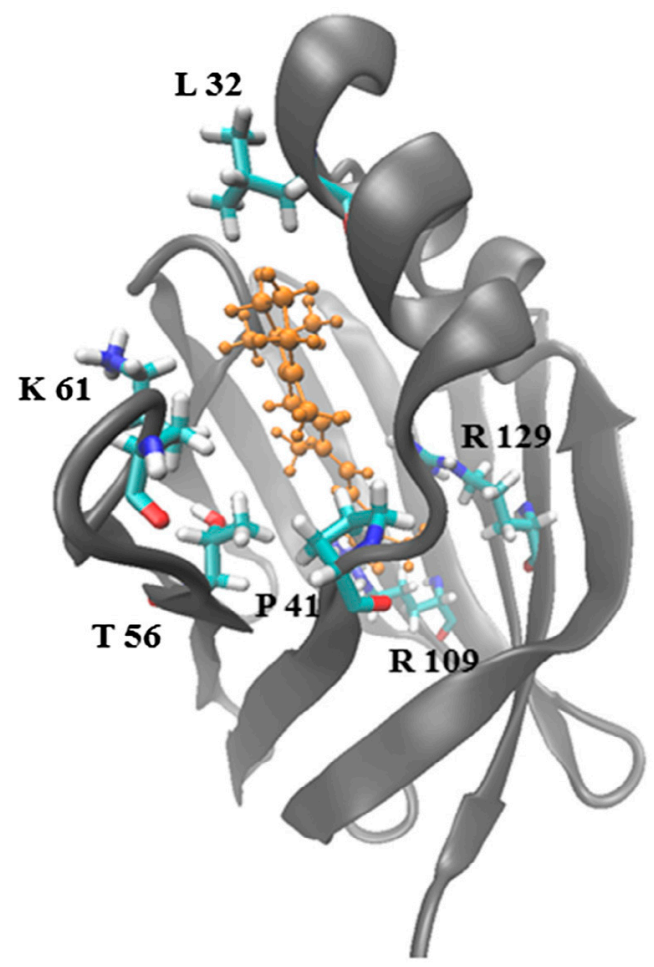

Figure 8. FABP5+RA $(t=150 \mathrm{~ns})$ showing the residues that are most important in RA ligand (shown in orange) to FABP5 binding according to pairwise per-residue MM-GBSA calculations.

CRABP-II, not in the smaller, less flexible FABP5. Many questions remain about ligand entry and exit from iLBPs (Xiao, Fan, Zhou, Lin, \& Yang, 2016). While steered MD has been used to guide ligands into and out of binding pockets, the observation of a spontaneous ligand reorientation is quite unusual. Long et al. (2009) report a series of random expulsion molecular dynamics simulations (REMD) of two oleate ligands or two

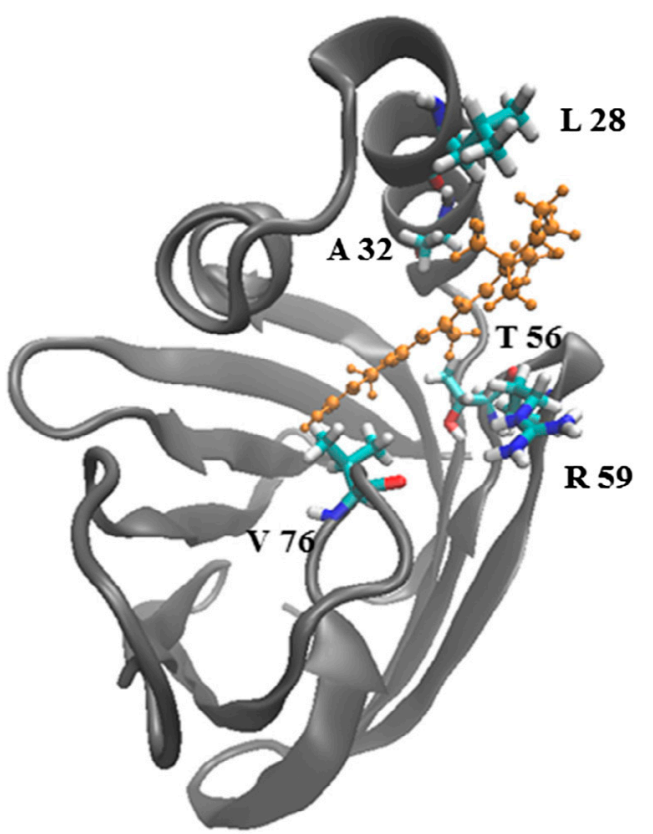

Figure 9. CRABP-II+RA $(t=150 \mathrm{~ns})$ showing the residues that are most important in RA ligand (shown in orange) to CRABP-II binding according to pairwise per-residue MM-GBSA calculations.

1-anilino-8-napthalene sulfonate ligands from the binding cavity of liver fatty acid binding protein, LFABP. They discovered three portals for ligand exit: the standard portal, a portal involving the $\beta \mathrm{G} / \beta \mathrm{H}$ loop, $\beta \mathrm{E} / \beta \mathrm{F}$ loop, $\mathrm{C}$-terminal end of $\alpha \mathrm{I}$ and $\mathrm{N}$-terminal end of $\alpha \mathrm{I} / \alpha \mathrm{II}$ loop, and the antiportal, a region on the opposite end of the $\beta$-barrel from the standard portal. Our results coupled with those of $\mathrm{Li}$ et al. and Long et al. are making it clear that the $\beta \mathrm{G} / \beta \mathrm{H}$ loop is likely more important than previously recognized in the mechanism of ligand binding and dynamics. 


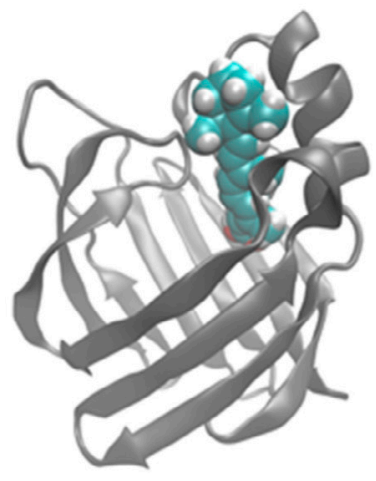

a) $50 \mathrm{~ns}$

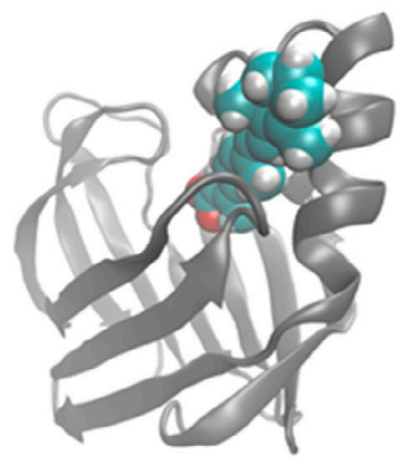

d) $200 \mathrm{~ns}$

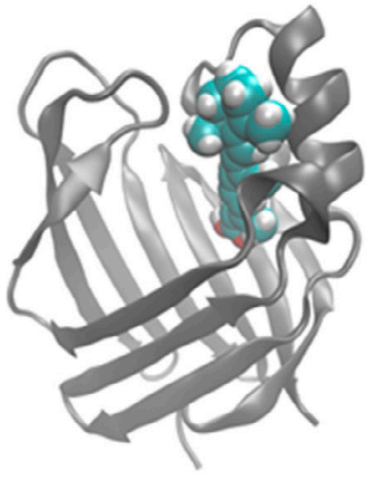

b) $100 \mathrm{~ns}$

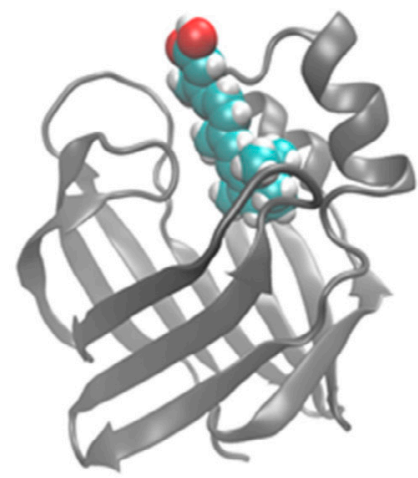

e) $230 \mathrm{~ns}$

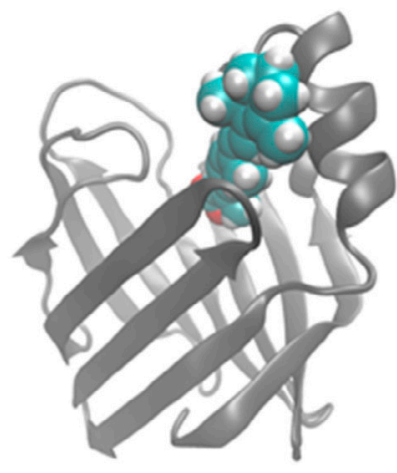

c) $150 \mathrm{~ns}$

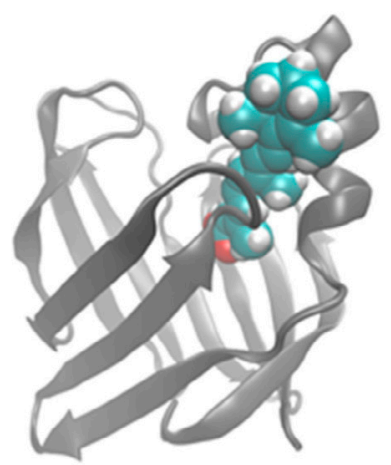

f) $250 \mathrm{~ns}$

Figure 10. CRABP-II+RA time evolution.

(a)

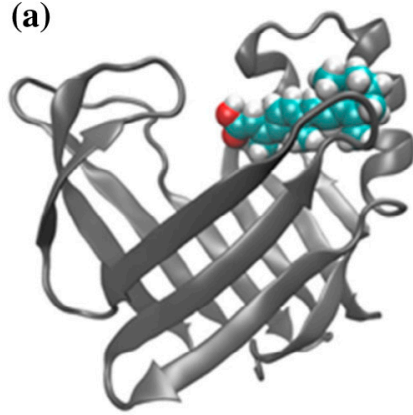

(b)

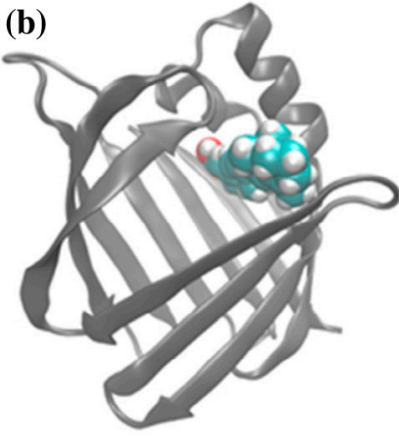

(c)

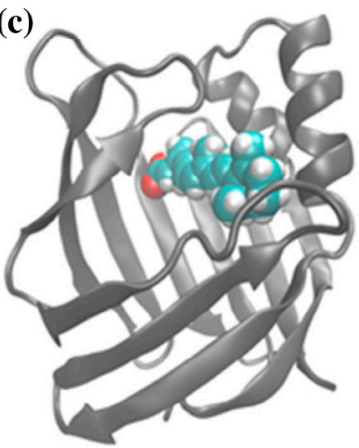

Figure 11. CRABP-II+RA (a) Initial simulation at $208 \mathrm{ns,} \mathrm{(b)} \mathrm{second} 300 \mathrm{~ns}$ simulation at $50 \mathrm{~ns}$, and (c) simulation with .15 M $\mathrm{NaCl}$ at $37.5 \mathrm{~ns}$ showing the RA ligand-oriented perpendicularly to the $\beta$-barrel. Unlike in the initial simulation, the RA returns to its original orientation with the carboxylic acid functional group in the interior of the protein as the simulation continues for both the second simulation and that with added salt.

To check the validity of our observations, we performed two additional $300 \mathrm{~ns}$ simualtions of CRABPII+RA: one using the same starting coordinates as the original simulation (referred to as Trajectory 2) and one with added salt to achieve $.15 \mathrm{M} \mathrm{NaCl}$. The Trajectory 2 experiment was conducted to see if there was an effect of the AMBER wall-clock-based random-number generator used in the generating the velocities for the simulation on the observed rotation. The added salt simulation was conducted because the literature on MD simulations of lipid binding proteins presents a mix of compuatational techniques for modeling the system. In our 
original design of this study, we followed the procedure of the recent study by Matsuoka et al. (2015) to provide a direct comparison of two FABPs (FABP3 in Matsuoka et al. and FABP5 in this study). Li et al. (2014) conducts their study of FABP4 in $.15 \mathrm{M} \mathrm{NaCl}$. Figure S9 shows the time dependence of the radius of gyration for all three CRABP-II+RA simulations. In all three simulations, the protein in closed more than it was in the apo simulation. In Trajectory 2 and the added salt simulation, CRABP-II spends even more time in the closed conformation than it did the original simulation, and the RA ligand does not completely reorient so that the carboxylic acid functional groups is in the bulk water. However, in both additional simulations, the ligand did orient sideways in the binding pocket as if it might flip into the solvent. It does not continue the rotation, but instead reorients so that the carboxylic acid functional group returns to the center of the protein. Figure 11 shows the sideways orientation of the RA ligand at various points in the each of the three simulation. Figure 11(a) shows the precursor to complete rotation that was observed in the original simulation. Figure 9(b) and (c) show that the ligand starts what could be the rotation out of the binding pocket. It does not, however, complete that rotation in either additional simulation. In all three cases, the $\beta \mathrm{G} / \beta \mathrm{H}$ loop is oriented away from the binding pocket and into the solvent.

\subsection{Trapped water molecules}

Water molecules are implicated in many important biological processes (de Beer, Vermeulen, \& Oostenbrink, 2010; Likic, Juranic, Macura, \& Prendergast, 2000; Nash, Saßmannshausen, Bozec, Birch, \& de Leeuw, 2017; Uroshlev et al., 2016). The crystal structures of FABP5 (pdb id 1B56) and CRABP-II (pdb id 2CBS) had water molecules present: 39 water molecules in the FABP5 crystal structure, 6 of which are within $4 \AA$ of the bound ligand, and 53 water molecules in the CRABP-II crystal structure, only 2 of which are within 4 $\AA$ of the bound ligand. These and other water molecules may be important in lipid recognition (Matsuoka et al., 2015). While the simulations presented here have shown that the mobility of the ligand and protein is much greater than a crystal structure can demonstrate, we also wanted to determine if there were water molecules with long residence times in the simulation and how those compared to the crystal structure. B-factor values for each water in all four simulations were calculated to determine the average distance fluctuations per water molecule. In general, water molecules had lower B-factor values in the FABP5 simulations than in the CRABP-II simulations. Therefore, the water molecules mimic the protein in that CRABP-II waters demonstrate more motion as did the CRABP-II protein. To determine which waters were trapped in a certain location, those with B-factors less than $18,000 \AA^{2}$ for FABP5 and $21,000 \AA^{2}$ for CRABP-II were defined as having a long residence time. This resulted in the identification of 12 trapped waters in FABP5 Only, 6 each in FABP5+RA and CRABP-II+RA, and 1 water molecule with a long residence time in the CRABP-II Only simulation. The large magnitude of the $\mathrm{B}$-factors for most water molecules indicates that they are very mobile throughout the simulation. The large binding cavity accommodates many water molecules, and these exchange with bulk waters regularly.

To determine where trapped water molecules were located and how long they were spending in that region, the distances between the center of mass of the identified trapped water and a point in the portal (residue Val 28 FABP5 and residue Gly 23 for CRABP-II), a point in the gap (residue 65 for FABP5 and residue 63 for CRABP-II), and for the holo simulations, the ligand center of mass were calculated. An example of the results from distance monitoring for water number 504 in FABP5 Only, one of 12 trapped water molecules in that simulation and the one with the 6th smallest B-factor of any water in the simulation, is shown in Figure 12. The water molecule is in roughly the same location from $\sim 120$ to $164 \mathrm{~ns}$ of the simulation. In Figure 13, water 504 is shown at 50,150 , and $250 \mathrm{~ns}$.

Two of the remaining trapped water molecules in the FABP5 Only simulation have a particularly long residence time. They enter the protein around $t=80 \mathrm{~ns}$ and remain inside throughout the remainder of the simulation. Snapshots showing their location are found in Figure 14. One of these water molecules is located within hydrogen-bond distance of FABP5 residue Gly

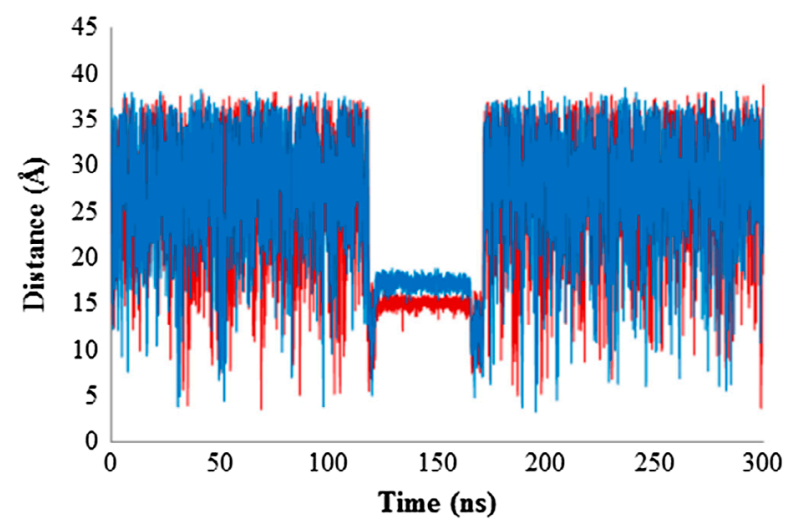

Figure 12. Distances from water center of mass of one trapped water molecule (\#504) in the FABP5 Only simulation to the $\mathrm{C} \alpha$ atom of residue Val 28 in the portal (red) and the $\mathrm{C} \alpha$ atom of residue Phe 63 in the gap (Blue). The region around $150 \mathrm{~ns}$ is where the water molecule has a long residence time inside the protein as shown in Figure 13. 


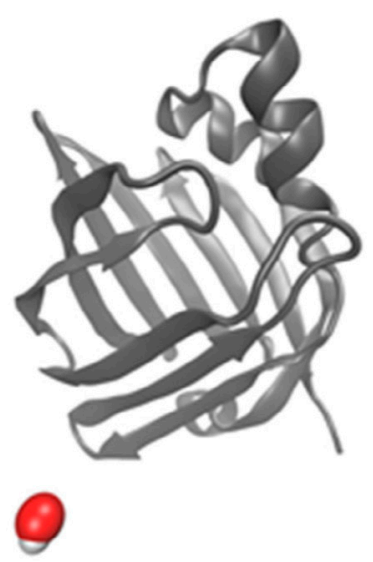

a) $50 \mathrm{~ns}$

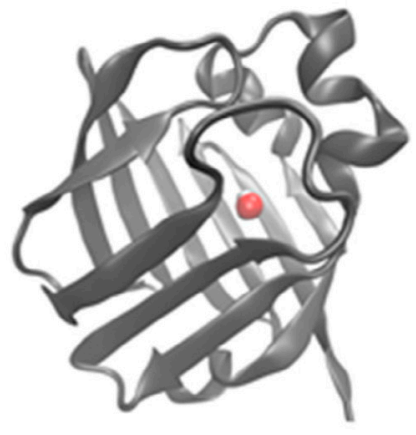

b) $150 \mathrm{~ns}$
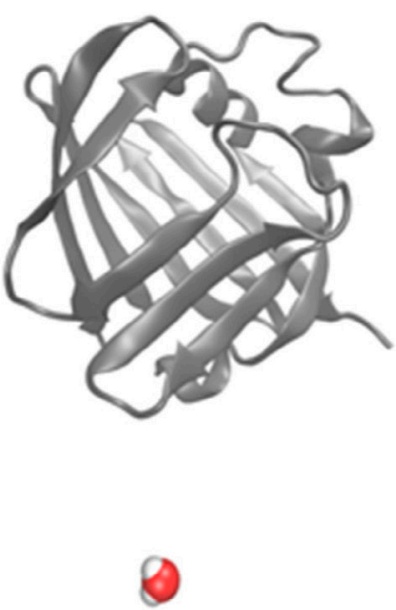

c) $250 \mathrm{~ns}$

Figure 13. A single water molecule that spends time trapped in protein around $150 \mathrm{~ns}$. Distance monitoring between select protein atoms and the water molecule center of mass is shown in Figure 12.

(a)

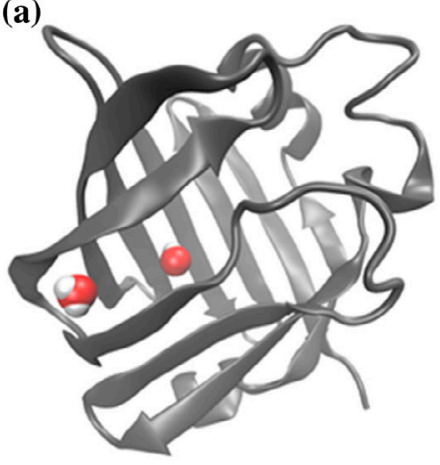

(b)

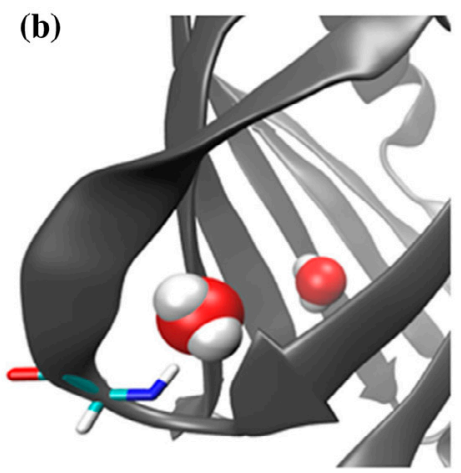

(c)

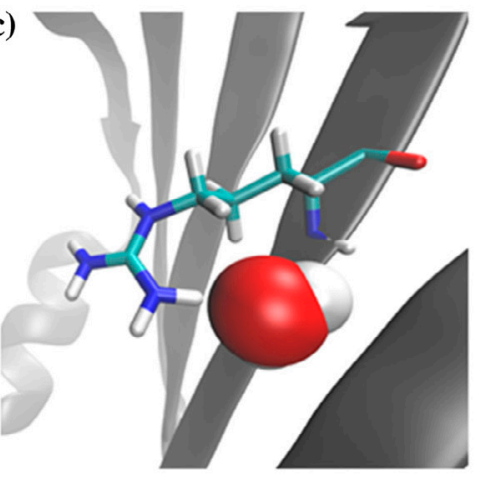

Figure 14. Snapshots of water molecules with lowest B-factor values in FABP5 Only at 150 ns. (a) Global view, (b) close up of water in Gap region interacting with residue Gly 70, and (c) close up of water between $\beta-\mathrm{G}$ and $\beta-\mathrm{H}$ and residue Arg 109.

70, which is near the turn between $\beta-\mathrm{D}$ and $\beta-\mathrm{E}$ (the gap).

A water molecule in this location has been observed in multiple iLBPs and is thought to be important in the folding mechanism of this class of proteins (Kaieda \& Halle, 2015; Kim, Ramanathan, \& Frieden, 1997; Likic et al., 2000; Ropson \& Frieden, 1992). The second water molecule with a long residence time is located between $\beta-\mathrm{G}$ and $\beta-\mathrm{H}$ and near residue Arg 109, an important residue for forming hydrogen bonds with water molecules in the FABP5 crystal structure. The remaining water molecules that have low B-factor values in the FABP5 Only simulation reside in the protein for less time, and many enter or exit the protein via the gap. In FABP5 crystal structures, Arg 129 and Tyr 131 are particularly important as locations for hydrogen bonding between the ligand and the protein (Armstrong et al.,
2014; Van Tilbeurgh et al., 1999). In the simulations presented here, the ligand is more mobile and appears to hydrogen bond more readily with Arg 109, a neighboring arginine residue, as mentioned earlier. In the FABP5 Only simulations, these potential hydrogen bonding locations are occupied by water molecules instead of a ligand. A snapshot of all 12 water molecules that become trapped at some point during the FABP5 Only simulation can be found in Figure S10 in the Supplementary Material. These results are consistent with those reported by Armstrong et al. who find a relatively large number of ordered water molecules in the crystal structure located in the top of the $\beta$-barrel near the $\alpha$-helices and the portal, and they suggest that this large number of ordered waters is indicative of the high enthalpic contribution to binding that is a feature of FABPs (Armstrong et al., 2014). 


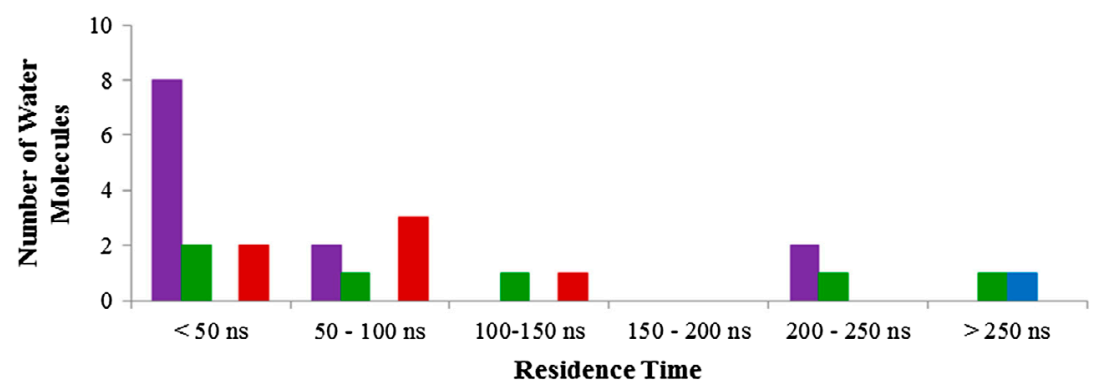

Figure 15. Trapped water molecules and their residence times in each simulation. FABP5 Only (purple) and FABP5+RA (green), CRABP-II Only (blue), and CRABPII+RA (red).

The FABP5+RA simulation shows half the number of trapped waters as FABP5 Only. The two water molecules with the lowest B-factor values are similar to those in FABP5 Only in that they enter the protein early in the simulation and they are located in the same positions: one is in the gap near the turn between $\beta-D$ and $\beta$-E and the other is between $\beta-G$ and $\beta-H$. Neither of these water molecules start inside the protein, but once they are in, they stay at these locations. The other four water molecules with lower B-factors are closer to the ligand, but they are primarily interacting with the protein residues, not the RA, and they are more mobile. Unlike the FABP5 Only simulation where several water molecules can be trapped in the ligand at the same time, usually only one or two trapped water molecules are inside the protein in the FABP5+RA simulation.

As mentioned earlier, the CRABP-II simulations have fewer trapped water molecules. Both CRABP-II Only and CRABP-II+RA have one trapped water that is located in the gap near the turn between $\beta$-D and $\beta$-E. Once again, Gly 68 (equivalent to FABP5 Gly 70) is important in holding this water molecule in the gap. That all four systems show this trapped water molecule and it is the only trapped water molecule in the CRABP-II Only simulation supports its importance in the structure of the iLBPs. CRABP-II+RA has five additional water molecules that have a measurable residence time, but like FABP5+RA, there are usually only one or two trapped water molecules in the interior of the protein at a time during the simulation. Around $63 \mathrm{~ns}$, one of the trapped water molecules is forming a hydrogen bond with the RA ligand. Otherwise, the trapped water molecules are interacting with the protein rather than the ligand. Kaieda and Halle find that most water molecules in the binding cavity of another FABP, rat intestinal FABP or rIFABP, exchange hydration sites in approximately $1 \mathrm{~ns}$, slower than that of bulk water, and that there are a few water molecules (2.6 for apo and 4.8 for palmitate-bound rIFABP) that are trapped in the protein for more than $100 \mathrm{~ns}$. To quantify the mobility, or lack thereof, of the trapped water molecules in the four simulations presented here, the residence time for each water molecules was determined and the number of trapped water molecules within a given range of residence times is shown in Figure 15. There are more trapped water molecules in holo CRABP-II than in apo CRABP-II, but the opposite is true for FABP5. However, the single trapped water molecule in apo CRABP-II has one of the longest residence times.

\section{Conclusions}

The work presented here provides insight into the challenges of designing small molecules that prevent retinoic acid from binding to FABP5 and activating the PPAR $\beta / \delta$ pathway. FABP5 is a smaller, less flexible ligand than CRABP-II. It might initially seem that the increased flexibility of CRABP-II would result in weaker binding of RA, but the opposite is true. CRABP-II binds RA more strongly than FABP5 does. The simulations presented here may provide some insight as to why that is the case. FABP5, like FABP4, (Li et al., 2014) spends more time in an open conformation in the holo form relative to the apo form than CRABP-II does. The CRABP-II protein closes significantly upon ligand binding as observed in three separate $300 \mathrm{~ns}$ simulations. Ligands that have the potential to block the FABP5/PPAR $\beta / \delta$ pathway should be designed to result in a more closed structure for FABP5 upon binding. Suggestions for accomplishing this task include varying the degree of saturation of fatty acids and improving hydrogen bonding or $\pi$-stacking opportunities between the ligand and the protein (Armstrong et al., 2014; Li et al., 2014). A significant challenge in predictive ligand design, though, is the growing recognition based on computational and solution-phase experiments that the protein and ligand are both far more mobile than crystal structures indicate (Hughes et al., 2012). The research presented here provides some insight into the origin and impacts of this mobility. Our observation of the rotation of the RA ligand out of the CRABPII binding pocket demonstrates that (a) the $\beta \mathrm{G} / \beta \mathrm{H}$ loop is more important in ligand entry and exit than previously 
thought, (b) the single definition of the portal as the separation of the $\beta E / \beta F$ loop and $\alpha$ II is insufficient to understanding portal dynamics, and (c) the hydrophobic interactions between the RA ligand and the protein are sufficiently strong to drive the ligand back into the binding pocket rather than escaping into the solvent. In fact, crystal structures would appear to indicate that hydrogen bonding is critical to strong ligand binding; however, our results show that persistent ligand-protein hydrogen bonding is not required for the ligand to remain bound. What may be more important is the location of a few important water molecules, including the ones located in the gap near the turn between $\beta-\mathrm{D}$ and $\beta-\mathrm{E}$ and between $\beta-\mathrm{G}$ and $\beta-\mathrm{H}$.

\section{Supplementary material}

Additional information about the protein sequence, atom labeling, and time-dependence of some the results presented in the manuscript is available free of charge via the Internet. Available online at https://doi.org/10.1080/ 07391102.2017.1337591.

\section{Acknowledgments}

The authors would like to thank Katie Doud for thoughtful conversations that instigated this project. This work was supported in part by the Molecular Education and Research Consortium in Undergraduate computational chemistRY (MERCURY), NSF CHE MRI Grant \#1662030 and the John Carroll University Colleran-Weaver Summer Undergraduate Research Fellowship.

\section{Disclosure statement}

No potential conflict of interest was reported by the authors.

\section{Funding}

This work was supported by the National Science Foundation [grant number 1662030] and the Colleran-Weaver Summer Undergraduate Research Fellowship program.

\section{ORCID}

Chrystal D. Bruce (D) http://orcid.org/0000-0001-9221-8960

\section{References}

Armstrong, E. H., Goswami, D., Griffin, P. R., Noy, N., \& Ortlund, E. A. (2014). Structural basis for ligand regulation of the fatty acid-binding protein 5 , peroxisome proliferatoractivated receptor beta/delta (FABP5-PPAR beta/delta) signaling pathway. Journal of Biological Chemistry, 289, 14941-14954. doi:10.1074/jbc.M113.514646

Atshaves, B. P., McIntosh, A. L., Storey, S. M., Landrock, K. K., Kier, A. B., \& Schroeder, F. (2010). High dietary fat exacerbates weight gain and obesity in female liver fatty acid binding protein gene-ablated mice. Lipids, 45, 97-110. doi:10.1007/s11745-009-3379-2

Ayers, S. D., Nedrow, K. L., Gillilan, R. E., \& Noy, N. (2007). Continuous nucleocytoplasmic shuttling underlies transcriptional activation of PPARgamma by FABP4. Biochemistry, 46, 6744-6752.

Balendiran, G. K., Schnutgen, F., Scapin, G., Borchers, T., Xhong, N., Lim, K., ... Sacchettini, J. C. (2000). Crystal structure and thermodynamic analysis of human brain fatty acid-binding protein. Journal of Biological Chemistry, 275, 27045-27054

de Beer, S. B. A., Vermeulen, N. P. E., \& Oostenbrink, C. (2010). The role of water molecules in computational drug design. Current Topics in Medicinal Chemistry, 10, 55-66. doi:10.2174/156802610790232288

Cao, H., Gerhold, K., Mayers, J. R., Wiest, M. M., Watkins, S. M., \& Hotamisligil, G. S. (2008). Identification of a lipokine, a lipid hormone linking adipose tissue to systemic metabolism. Cell, 134, 933-944. doi:10.1016/j.cell.2008.07.048

Chen, J., Wang, J., \& Zhu, W. (2014). Binding modes of three inhibitors 8CA, F8A and I4A to A-FABP studied based on molecular dynamics simulation. PLoS One, 9, e99862. doi:10.1371/journal.pone.0099862

De Leon, M., Welcher, A. A., Nahin, R. H., Liu, Y., Ruda, M. A., Shooter, E. M., \& Molina, C. A. (1996). Fatty acid binding protein is induced in neurons of the dorsal root ganglia after peripheral nerve injury. Journal of Neuroscience Research, 44, 283-292. doi:10.1002/(SICI)10974547(19960501)44:3<283::AID-JNR9>3.3.CO;2-R

Dong, D., Ruuska, S. E., Levinthal, D. J., \& Noy, N. (1999). Distinct roles for cellular retinoic acid-binding proteins I and II in regulating signaling by retinoic acid. Journal of Biological Chemistry, 274, 23695-23698. doi:10.1074/ jbc.274.34.23695

Ferrolino, M. C., Zhuravleva, A., Budyak, I. L., Krishnan, B., \& Gierasch, L. M. (2013). Delicate balance between functionally required flexibility and aggregation risk in a $\beta$-rich protein. Biochemistry, 52, 8843-8854. doi:10.1021/ bi4013462

Genheden, S., \& Ryde, U. (2015). The MM/PBSA and MM/ GBSA methods to estimate ligand-binding affinities. Expert Opinion on Drug Discovery, 10, 449-461. doi:10.1517/ 17460441.2015.1032936.

Gillilan, R. E., Ayers, S. D., \& Noy, N. (2007). Structural basis for activation of fatty acid-binding protein 4. Journal of Molecular Biology, 372, 1246-1260. doi:10.1016/ j.jmb.2007.07.040

Glatz, J. F. C. (2015). Lipids and lipid binding proteins: A perfect match. Prostaglandins, Leukotrienes and Essential Fatty Acids (PLEFA), 93, 45-49. doi:10.1016/j.plefa.2014.07.011

Hohoff, C., Börchers, T., Rüstow, B., Spener, F., \& Van Tilbeurgh, H. (1999). Expression, purification, and crystal structure determination of recombinant human epidermaltype fatty acid binding protein. Biochemistry, 38, 12229 12239. doi:10.1021/bi990305u

Hughes, T. S., Chalmers, M. J., Novick, S., Kuruvilla, D. S., Chang, M. R., Kamenecka, T. M., ... Kojetin, D. J. (2012). Ligand and Receptor dynamics contribute to the mechanism of graded PPAR $\gamma$ agonism. Structure, 20, 139-150. doi:10.1016/j.str.2011.10.018

Humphrey, W., Dalke, A., \& Schulten, K. (1996). VMD: Visual molecular dynamics. Journal of Molecular Graphics, 14, 33-38. 
Kaieda, S., \& Halle, B. (2015). Time scales of conformational gating in a lipid-binding protein. Journal of Physical Chemistry $B, \quad 119, \quad 7957-7967$. doi:10.1021/acs.jpcb. $5 \mathrm{~b} 03214$

Kim, K., Ramanathan, R., \& Frieden, C. (1997). Intestinal fatty acid binding protein: A specific residue in one turn appears to stabilize the native structure and be responsible for slow refolding. Protein Science, 6, 364-372.

Levin, L. B.-A., Ganoth, A., Amram, S., Nachliel, E., Gutman, M., \& Tsfadia, Y. (2010). Insight into the interaction sites between fatty acid binding proteins and their ligands. Journal of Molecular Modeling, 16, 929-938. doi:10.1007/ s00894-009-0599-6

Li, Y., Li, X., \& Dong, Z. (2014). Concerted dynamic motions of an FABP4 model and its ligands revealed by microsecond molecular dynamics simulations. Biochemistry, 53, 6409-6417.

Likic, V. A., Juranic, N., Macura, S., \& Prendergast, F. G. (2000). A 'structural' water molecule in the family of fatty acid binding proteins. Protein Science, 9, 497-504.

Liu, J.-W., Almaguel, F. G., Bu, L., De Leon, D. D., \& De Leon, M. (2008). Expression of E-FABP in PC12 cells increases neurite extension during differentiation: Involvement of n-3 and n-6 fatty acids. Journal of Neurochemistry, 106, 2015-2029. doi:10.1111/j.1471-4159.2008.05507.x

Long, D., Mu, Y., \& Yang, D. (2009). Molecular dynamics simulation of ligand dissociation from liver fatty acid binding protein. PLoS One, 4, e6081. doi:10.1371/journal.pone. 0006081

Lucke, C., Gutierrez-Gonzalez, L. H., \& Hamilton, J. A. (2003). Intracellular lipid binding proteins: Evolution, structure, and ligand binding. Weinheim: Wiley-VCH.

Marr, E., Tardie, M., Carty, M., Brown Phillips, T., Wang, I. K., Soeller, W., ... Karam, G. (2006). Expression, purification, crystallization and structure of human adipocyte lipidbinding protein (aP2). Acta Crystallographica Section F Structural Biology and Crystallization Communications, 62, 1058-1060. doi:10.1107/S1744309106038656

Matsuoka, S., Sugiyama, S., Matsuoka, D., Hirose, M., Lethu, S., Ano, H., ... Murata, M. (2015). Water-mediated recognition of simple alkyl chains by heart-type fatty-acid-binding protein. Angewandte Chemie International Edition, 54, 1508-1511. doi:10.1002/anie.201409830

Matsuoka, D., Sugiyama, S., Murata, M., \& Matsuoka, S. (2015). Molecular dynamics simulations of heart-type fatty acid binding protein in apo and holo forms, and hydration structure analyses in the binding cavity. The Journal of Physical Chemistry B, 119, 114-127. doi:10.1021/ jp510384f

Miller, B. R., McGee, T. D., Swails, J. M., Homeyer, N., Gohlke, H., \& Roitberg, A. E. (2012). MMPBSA.py: An efficient program for end-state free energy calculations. Journal of Chemical Theory and Computation, 8, 3314-3321. doi:10.1021/ct300418h

Mueller, R. (2016). PPAR $\beta / \delta$ in human cancer. Biochimie, Ahead of Print. doi:10.1016/j.biochi.2016.10.019

Nash, A., Saßmannshausen, J., Bozec, L., Birch, H. L., \& de Leeuw, N. H. D. (2017). Computational study of glucosepane-water and hydrogen bond formation: An electron topology and orbital analysis. Journal of Biomolecular Structure and Dynamics, 35, 1127-1137. doi:10.1080/ 07391102.2016.1172026

Newberry, E. P., Xie, Y., Kennedy, S., Han, X., Buhman, K. K., Luo, J., ... Davidson, N. O. (2003). Decreased hepatic triglyceride accumulation and altered fatty acid uptake in mice with deletion of the liver fatty acid-binding protein gene. Journal of Biological Chemistry, 278, 51664-51672. doi:10.1074/jbc.M309377200

Newberry, E. P., Xie, Y., Kennedy, S. M., Luo, J., \& Davidson, N. O. (2006). Protection against Western diet-induced obesity and hepatic steatosis in liver fatty acid-binding protein knockout mice. Hepatology, 44, 1191-1205. doi:10.1002/ hep. 21369

Owada, Y. (2008). Fatty acid binding protein: Localization and functional significance in the brain. Tohoku Journal of Experimental Medicine, 214, 213-220. doi:10.1620/tjem.214.213

Peters, J. M., Gonzalez, F. J., \& Müller, R. (2015). Establishing the role of PPAR $\beta / \delta$ in carcinogenesis. Trends in endocrinology and metabolism: TEM, 26, 595-607. doi:10.1016/j.tem.2015.09.004

Roe, D. R., Cheatham, T. E., \& Cheatham, I. (2013). PTRAJ and CPPTRAJ: Software for processing and analysis of molecular dynamics trajectory data. Journal of Chemical Theory and Computation, 9, 3084-3095.

Ropson, I. J., \& Frieden, C. (1992). Dynamic NMR spectral analysis and protein folding: Identification of a highly populated folding intermediate of rat intestinal fatty acid-binding protein by $19 \mathrm{~F}$ NMR. Proceedings of the National Academy of Sciences, 89, 7222-7226. doi:10.1073/ pnas.89.15.7222

Ruskamo, S., Yadav, R. P., Sharma, S., Lehtimäki, M., Laulumaa, S., Aggarwal, S., ... Kursula, P. (2014). Atomic resolution view into the structure-function relationships of the human myelin peripheral membrane protein P2. Acta Crystallographica Section D Biological Crystallography, 70, 165-176. doi:10.1107/S1399004713027910

Salomon-Ferrer, R., Götz, A. W., Poole, D., Le Grand, S., \& Walker, R. C. (2013). Routine microsecond molecular dynamics simulations with AMBER on GPUs. 2. Explicit solvent particle Mesh Ewald. Journal of Chemical Theory and Computation, 9, 3878-3888. doi:10.1021/ct400314y

Schaap, F. G., Van der Vusse, G. J., \& Glatz, J. F. C. (2002). Evolution of the family of intracellular lipid binding proteins in vertebrates. Molecular and Cellular Biochemistry, 239, 69-77.

Schug, T. T., Berry, D. C., Shaw, N. S., Travis, S. N., \& Noy, N. (2007a). Dual transcriptional activities underlie opposing effects of retinoic acid on cell survival. Cell, 129, 723-733. doi:10.1016/j.cell.2007.02.050

Schug, T. T., Berry, D. C., Shaw, N. S., Travis, S. N., \& Noy, N. (2007b). Opposing effects of retinoic acid on cell growth result from alternate activation of two different nuclear receptors. Cell, 129, 723-733.

Schug, T. T., Berry, D. C., Toshkov, I. A., Cheng, L., Nikitin, A. Y., \& Noy, N. (2008). Overcoming retinoic acid-resistance of mammary carcinomas by diverting retinoic acid from PPAR beta/delta to RAR. Proceedings of the National Academy of Sciences, 105, 7546-7551. doi:10.1073/ pnas.0709981105

Storch, J., \& Thumser, A. E. (2010). Tissue-specific functions in the fatty acid-binding protein family. Journal of Biological Chemistry, 285, 32679-32683. doi:10.1074/jbc.R110. 135210

Sussman, F., \& de Lera, A. R. (2005). Ligand recognition by RAR and RXR receptors: Binding and selectivity. Journal of Medicinal Chemistry, 48, 6212-6219. doi:10.1021/jm050285w

Tan, N.-S., Shaw, N. S., Vinckenbosch, N., Liu, P., Yasmin, R., Desvergne, B., ... Noy, N. (2002). Selective cooperation between fatty acid binding proteins and peroxisome proliferator-activated receptors in regulating transcription. 
Molecular and Cellular Biology, 22, 5114-5127. doi:10.1128/MCB.22.14.5114-5127.2002

Uroshlev, L. A., Kulakovskiy, I. V., Esipova, N. G., Tumanyan, V. G., Rahmanov, S. V., \& Makeev, V. J. (2016). Role of structural water for prediction of cation binding sites in apoproteins. Journal of Biomolecular Structure and Dynamics, 1-12. doi:10.1080/07391102.2016.1273136

Van Tilbeurgh, H., Hohoff, C., Borchers, T., \& Spener, F. (1999). Expression, purification, and crystal structure determination of recombinant human epidermal-type fatty acid binding protein. Biochemistry, 38, 12229-12239.

Xiao, T., Fan, J.-S., Zhou, H., Lin, Q., \& Yang, D. (2016). Local unfolding of fatty acid binding protein to allow ligand entry for binding. Angewandte Chemie International Edition, 55, 6869-6872. doi:10.1002/anie.201601326
Xie, Y., Newberry, E. P., Kennedy, S. M., Luo, J., \& Davidson, N. O. (2009). Increased susceptibility to diet-induced gallstones in liver fatty acid binding protein knockout mice. Journal of Lipid Research, 50, 977-987. doi:10.1194/ jlr.M800645-JLR200

Xu, Z., Bernlohr, D. A., \& Banaszak, L. J. (1993). The adipocyte lipid-binding protein at 1.6-A resolution. Crystal structures of the apoprotein and with bound saturated and unsaturated fatty acids. Journal of Biological Chemistry, 268, 7874-7884.

Zhou, Y., Nie, T., Zhang, Y., Song, M., Li, K., Ding, M., ... $\mathrm{Xu}, \mathrm{Y}$. (2016). The discovery of novel and selective fatty acid binding protein 4 inhibitors by virtual screening and biological evaluation. Bioorganic \& Medicinal Chemistry, 24, 4310-4317. doi:10.1016/j.bmc.2016.07.022 